\title{
Merging of the Dirac points in electronic artificial graphene
}

\author{
J. Feilhauer, ${ }^{1,2,-\circledast}$ W. Apel, ${ }^{1}$ and L. Schweitzer ${ }^{1}$ \\ ${ }^{1}$ Physikalisch-Technische Bundesanstalt (PTB), Braunschweig, Germany \\ ${ }^{2}$ Institute of Electrical Engineering, Slovak Academy of Sciences, 84104 Bratislava, Slovakia
}

(Dated: August 3, 2018)

\begin{abstract}
Theory predicts that graphene under uniaxial compressive strain in an armchair direction should undergo a topological phase transition from a semimetal into an insulator. Due to the change of the hopping integrals under compression, both Dirac points shift away from the corners of the Brillouin zone towards each other. For sufficiently large strain, the Dirac points merge and an energy gap appears. However, such a topological phase transition has not yet been observed in normal graphene (due to its large stiffness) neither in any other electronic system. We show numerically and analytically that such a merging of the Dirac points can be observed in electronic artificial graphene created from a two-dimensional electron gas by application of a triangular lattice of repulsive antidots. Here, the effect of strain is modeled by tuning the distance between the repulsive potentials along the armchair direction. Our results show that the merging of the Dirac points should be observable in a recent experiment with molecular graphene.
\end{abstract}

PACS numbers: 73.20.At, 73.22.Pr

\section{INTRODUCTION}

In the past decade, graphene $\frac{1}{-}$ became one of the most studied materials due to its unusual properties that are attractive from the perspective of basic physics as well as from that of a future technology. In graphene, the two-dimensional hexagonal lattice of carbon atoms provides for a gapless electronic bandstructure which consist of two bands touching each other at two Dirac points located at the independent corners of the hexagonal Brillouin zone. The Fermi energy of natural graphene lies in the vicinity of the Dirac points, where the bands have linear dispersion and conical shape. Therefore at low energies, the bandstructure of graphene can be modeled by two Dirac cones and electrons behave as massless Dirac particles with a valley isospin.

After the discovery of graphene, there has been a significant effort ${ }^{2-11}$ to create artificial systems with hexagonal symmetry that mimic the unique properties of Dirac quasiparticles. The successful experimental realization of Dirac quasiparticles was reported in systems of cold atoms in hexagonal optical lattices,,$\frac{3}{2}$ in photonic crystals created by a hexagonal array of optical waveguides $\stackrel{4,5}{,}$ and in two-dimensional electron gases with hexagonal superlattices $\stackrel{\underline{6}-10}{=}$ The main advantage of these artificial systems is that one can control and tune the system parameters (i.e., the lattice constant and hopping parameters) independently. That makes it possible to study features that are not observable in normal graphene, e.g., the extremely large pseudomagnetic fields induced by strain, Kekulé lattices, edge states, etc.

One of the interesting possibilities in artificial graphene is to induce lattice anisotropy by tuning the hopping parameters between the lattice sites. The theory based on the nearestneighbor tight-binding approximation predicts ${ }^{12-16}$ that by changing the hopping parameters, both Dirac cones are shifted away from the corners of the Brillouin zone and become elliptical instead of circular. With increasing anisotropy, the Dirac cones merge and an energy gap is created. This causes a topological phase transition ${ }^{15,17}$ where the system turns from a semimetal into an insulator. At the critical point of this merg- ing, the energy bands touch only in a single point with an unusual dispersion relation in its vicinity $\frac{16}{=}$ In one direction in $\mathrm{k}$-space, the dispersion is linear as in the standard Dirac cone but in the perpendicular direction, the dispersion is quadratic as in the case of massive electrons.

In normal graphene, lattice anisotropy could be induced by application of uniaxial strain that changes the distance between the lattice sites. But the values of strain that would lead to the topological phase transition are unrealistically large owing to the large graphene stiffness $\frac{13}{}$ Thus, merging of the Dirac points was demonstrated experimentally in systems of cold atoms ${ }^{3,18}$ and microwave photonic crystals ${ }^{4}$ where the distance between lattice sites can be varied much more than in normal graphene. In spite of some efforts, $\underline{\underline{6}}$ a merging of the Dirac points has not yet been confirmed in any electronic system.

Here, we show analytically and numerically that a merging of Dirac points appears also in electronic artificial graphene under uniaxial compressive 'strain'. We study artificial graphene created in a two-dimensional electron gas modulated by a triangular lattice of repulsive potentials, e.g., repulsive molecules on a copper surface $\frac{6.7}{}$ or a muffin-tin potential of antidots in a semiconductor heterostructure. ${ }^{8-10}$ In such systems, the electrons are repelled into the space between the antidots which leads to hexagonal symmetry. The lowest energy bands of artificial graphene are similar to the two-level tight-binding bandstructure of natural graphene although the electrons are not bound to any attractive potential. The lattice constant of artificial graphene can be orders of magnitude larger than in natural graphene and, moreover, the position of the artificial lattice sites can be easily tuned. Strain is simulated by tuning the distance between the repulsive triangular potentials along the armchair direction. When the antidot lattice is stretched, the Dirac points are shifted away from the corners of the Brillouin zone into its interior, but they always exist. On the other hand, with increasing compressive strain, both Dirac points are moving along the edge of the Brillouin zone towards each other until they merge with a dispersion as described above. For larger compression, a bulk gap is cre- 
ated.

We believe that our theoretical results could be verified experimentally in the recently created molecular graphene,, .7 where a two-dimensional electron gas on the $\mathrm{Cu}$ surface is modulated by a triangular antidot lattice of repulsive molecules (CO or coronene). Here, the presence of a Dirac energy spectrum is proven by measurements of the density of states (DOS) which is similar to typical graphene DOS with the zero value at the energy corresponding to the Dirac point. The authors of [6] performed an experiment where the artificial graphene was elongated in the armchair direction by about $30 \%$. They observed no gap opening in the DOS and that is consistent with our numerical results for the case of streched artificial graphene. Nevertheless, our numerical calculations of the DOS show that the molecular graphene with coronene ${ }^{7}$ is a very promising candidate to observe the merging of the Dirac points and related gap opening in the DOS. This should occur, however, for a lattice compression of about $25 \%$, and that is technologically feasible.

This paper is organized as follows. In the second section, we review the results of the analytical tight-binding calculations that describe the merging of the Dirac points in the hexagonal lattice with the hopping matrix anisotropy. In the third section, we define mathematically the model of artificial graphene and discuss the effect of uniaxial strain. Then we describe the technique that we used to calculate the numerical results. The fourth section contains our numerical results of the bandstructure for artificial graphene under uniaxial strain. The calculations show that the merging of the Dirac points occurs in the artificial graphene under compressive strain in the armchair direction. The fifth section shows the analytical calculation of the low-energy bandstructure of strained artificial graphene in the nearly-free electron approximation, which is valid for weak potentials. The analytical formulas confirm the merging of the Dirac points in the compressed artificial graphene and are in a good agreement with the numerical results. In the sixth section, we discuss the realizability of our calculations in real experimental situations and we conclude that the merging of the Dirac points should be observable in the recent experiment with molecular graphene with coronene $?^{?}$

\section{MERGING OF THE DIRAC POINTS: TIGHT-BINDING MODEL DESCRIPTION}

In this section, we review the results of the nearest-neighbor tight-binding calculations ${ }^{12-16}$ concerning the bandstructure of a hexagonal lattice with anisotropy in the hopping matrix elements. We examine the positions of the Dirac points in the Brillouin zone.

Normal graphene consists of a triangular Bravais lattice with a pair of carbon atoms located in its primitive cell. The lattice vectors are

$$
\mathbf{b}_{1}=\frac{L}{2} \mathbf{e}_{x}-\frac{\sqrt{3} L}{2} \mathbf{e}_{y}, \quad \mathbf{b}_{2}=\frac{L}{2} \mathbf{e}_{x}+\frac{\sqrt{3} L}{2} \mathbf{e}_{y}
$$

where $L$ is a lattice constant and $L / \sqrt{3}$ is the distance be- a)
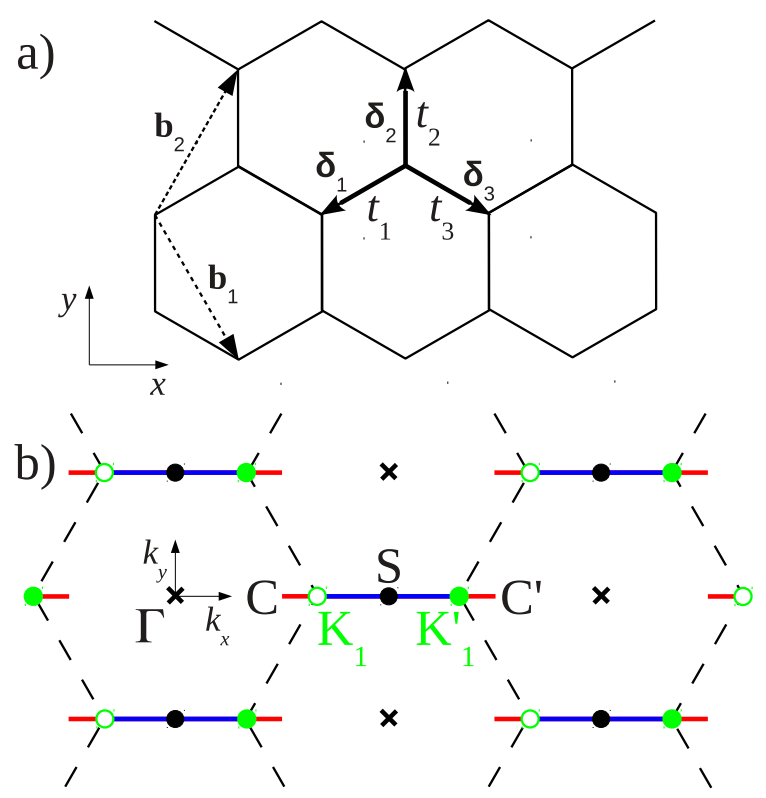

FIG. 1: (a) Model of the hexagonal lattice of graphene. Each lattice site has three nearest-neighbors with the locations given by the vectors $\delta_{i}$ (see Eq 3) and corresponding hopping parameters $t_{i}$. (b) The repeated Brillouin zone scheme of graphene. The Brillouin zone contains a symmetry point $\Gamma$ and a pair of independent corners $K$ (green empty circles) and K' (green full circles) which are periodically repeated around the reciprocal lattice points (black crosses). When $t_{1}=t_{3}=t$ and $t_{2}=t^{\prime}$, the Dirac points are located on a line $\mathrm{CC}^{\prime}$. For $0<t^{\prime} / t \leq 1$, the Dirac points are located inside the Brillouin zone (red lines) and for $1<t^{\prime} / t \leq 2$ the Dirac points lie at the edge (blues lines). For $t^{\prime} / t=2$ the Dirac points merge at $\mathrm{S}$ (black dots).

tween neighboring carbon atoms. The corresponding reciprocal lattice is triangular as well with a hexagonal Brillouin zone with the side length $4 \pi /(3 L)$. In the nearest-neighbor tight-binding approximation, each carbon atom possesses one localized electronic state that slightly overlaps with its three nearest neighbors. These overlaps are in general characterized by three hopping parameters $t_{1}, t_{2}, t_{3}$ (see Fig. 11a)). In the isotropic case, all hopping parameters are equal. If we take the hopping anisotropy in armchair direction, we get $t_{1}=t_{3} \neq t_{2}$. Then, the bandstructure can be written in the form

$$
\epsilon(\mathbf{k})= \pm\left|t e^{i \mathbf{k} \cdot \boldsymbol{\delta}_{1}}+t^{\prime} \beta e^{i \mathbf{k} \cdot \boldsymbol{\delta}_{2}}+t e^{i \mathbf{k} \cdot \boldsymbol{\delta}_{3}}\right|,
$$

where we have defined $t_{1}=t_{3}=t, t_{2}=t^{\prime}$ and

$$
\left\{\boldsymbol{\delta}_{1}, \boldsymbol{\delta}_{2}, \boldsymbol{\delta}_{3}\right\}=\frac{1}{3}\left\{-\mathbf{b}_{1}-2 \mathbf{b}_{2}, \mathbf{b}_{2}-\mathbf{b}_{1}, 2 \mathbf{b}_{1}+\mathbf{b}_{2}\right\},
$$

are the vectors connecting an atom with its neighbors.

The bandstructure (2) consists of two bands that touch at zero energy at the Dirac points. We obtain their position in the reciprocal space by solving $\epsilon(\mathbf{k})=0$ which implies the conditions

$$
\cos \left(\frac{\sqrt{3}}{2} L k_{y}^{D}\right)= \pm 1, \quad \cos \left(\frac{1}{2} L k_{x}^{D}\right)=\mp \frac{t^{\prime}}{2 t} .
$$


The conditions (4) give two independent Dirac points per Brillouin zone which are periodically repeated through the whole reciprocal space. To study the evolution of the Dirac points with increasing hopping anisotropy, it is more convenient to use the repeated Brillouin zone scheme and to focus on the Dirac points $\mathrm{D}_{1}$ and $\mathrm{D}_{2}$ located at

$$
\begin{array}{r}
k_{y}^{\mathrm{D}_{1}}=k_{y}^{\mathrm{D}_{2}}=0, \\
k_{x}^{\mathrm{D}_{1}}=\frac{2}{L} \arccos \left(-\frac{t^{\prime}}{2 t}\right), \\
k_{x}^{\mathrm{D}_{2}}=\frac{4 \pi}{L}-k_{x}^{\mathrm{D}_{1}} .
\end{array}
$$

For the case of hopping anisotropy in the armchair direction, the position of the Dirac points in reciprocal space is restricted to the $k_{x}$ axis. Moreover, the positions of $\mathrm{D}_{1}$ and $\mathrm{D}_{2}$ are symmetric around the point $\mathrm{S}$ at $k_{x}=2 \pi / L$. Therefore, in the following text, we discuss mostly only the position of $\mathrm{D}_{1}$.

The position and existence of Dirac points is given by the value of the anisotropy parameter $t^{\prime} / t$. Firstly, if $0<t^{\prime} / t \leq$ 1 , the Dirac point $\mathrm{D}_{1}$ lies inside the Brillouin zone (red lines in Fig. 1(b)) and the lowest value of $k_{x}^{\mathrm{D}_{1}}$ is limited by the value $\pi / L$ for $t^{\prime} / t=0$ (point C). For $t^{\prime} / t=1$ (isotropic lattice), the Dirac point lies exactly at the corner of the Brillouin zone $\mathrm{K}$ with $k_{x}^{\mathrm{D}_{1}}=4 \pi / 3 L$. Secondly, if $1<t^{\prime} / t \leq 2$ the Dirac point is located at the edge of the Brillouin zone (blue lines in Fig. 1(b)) and for the critical point $t^{\prime} / t=2$, both $\mathrm{D}_{1}$ and $\mathrm{D}_{2}$ merge at the point $\mathrm{S}$. Thirdly, for $2<t^{\prime} / t$, Dirac points no longer exist and an energy gap opens.

The hopping anisotropy discussed above could be achieved in normal graphene by applying an uniaxial strain in the armchair direction. When the graphene sample is stretched, the interatomic distance $\left|\boldsymbol{\delta}_{2}\right|$ increases. Then, the overlap of electron wavefunctions is suppressed and the hopping parameter $t_{2}$ decreases. This corresponds to the situation where $t^{\prime} / t<1$. On the other hand, when the sample is compressed, the interatomic distance $\left|\boldsymbol{\delta}_{2}\right|$ decreases and $t_{2}$ increases. Then $t^{\prime} / t>1$ and it increases with increasing compression. In principle, it could be possible to observe a merging of the Dirac points (for $t^{\prime} / t=2$ ) in graphene, but, unfortunately, the mechanical stiffness of graphene is very large $\frac{13}{3}$ and the sufficient compression to obtain $t^{\prime} / t=2$ is unreachable.

To observe a merging of the Dirac points, one has to arrange a system with hexagonal symmetry where it is possible to tune the hopping parameters in a wider interval than in normal graphene. This was already shown in a lattice of cold atoms $\stackrel{3,18}{ }$ or a photonic crystal,,$\frac{4}{+}$ but not yet for any electronic system. Our aim is to study a merging of the Dirac points in electronic artificial graphene described in the next section.

\section{ARTIFICIAL GRAPHENE UNDER UNIAXIAL STRAIN}

There have been several theoretical and experimental attempts to create an electronic artificial graphene. Most of them are based on a nanopatterning of a two-dimensional electron gas by an external periodic potential. One possibility
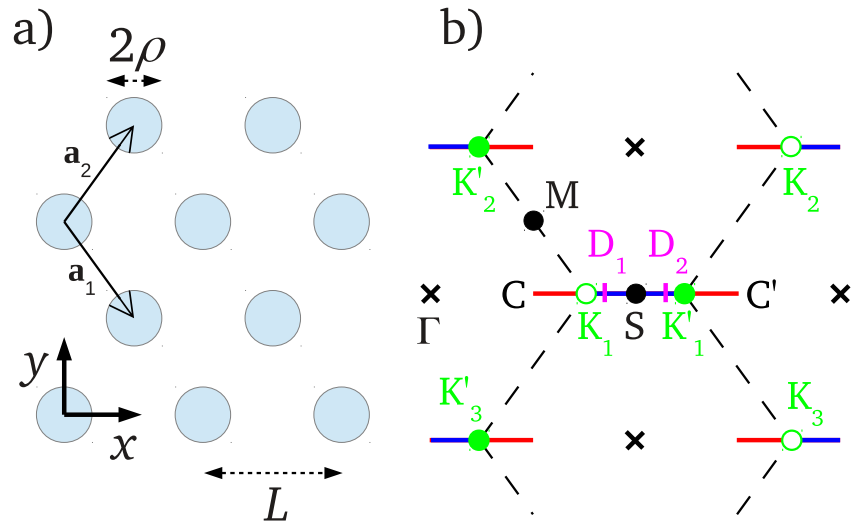

FIG. 2: (a) Triangular lattice of antidot potential spanned between the vectors $\mathbf{a}_{1}$ and $\mathbf{a}_{2}$. The antidot radius is $\rho / L=0.2$ and the lattice is compressed in the $y$ (armchair) direction with $\alpha=1.25$. (b) The corresponding reciprocal lattice (crosses) is triangular as well and it is strained in the horizontal direction. In general, the first Brillouin zone is an irregular hexagon and we use the repeated Brillouin zone scheme (dashed lines). Because the antidot lattice is compressed, the Dirac points $D_{1}$ and $D_{2}$ are shifted away from the corners $K_{1}$ and $K_{1}^{\prime}$ of the Brillouin zone towards the point $\mathrm{S}$.

is to introduce a hexagonal lattice of potential wells, which was performed lithographically in a GaAs heterostructure. $?$ In the present paper, we study an artificial graphene created from a two-dimensional electron gas by application of a repulsive triangular potential of antidots $V(\mathbf{r})$ (see Fig. 2(a)). This kind of artificial graphene was extensively studied ${ }^{8,19-23}$ and it captures the main features of the experiments with molecular graphene, which is a two-dimensional electron gas on the copper surface modulated by the repulsive molecules of $\mathrm{CO}^{\underline{6}}$ and coronene $?$

It can be shown ${ }^{8}$ that the wavefunctions of the electrons from the two lowest energy bands are localized around the centers of the triangles formed by the antidots and thus form a hexagonal lattice. Consequently, the two lowest energy bands of artificial graphene are very similar to those of normal graphene. Namely, there is a pair of Dirac points with linear dispersion in their vicinity.

The effect of strain discussed in the previous section can be easily achieved in this artificial graphene by prolonging or reducing the dimension of the triangular antidot lattice in the armchair direction. This consequently changes the distances in the underlying hexagonal electron lattice.

\section{A. Trigonal antidot potential under uniaxial strain}

The antidot potential $V(\mathbf{r})$ consists of repulsive circles with radius $\rho$ ordered on a triangular lattice with lattice constant $L$ and reads

$$
V(\mathbf{r})=\mathcal{V} \sum_{\mathbf{R}} \Theta(\rho-|\mathbf{r}-\mathbf{R}|),
$$

where $\mathcal{V}$ is the potential strength, $\Theta$ is the Heaviside step function and $\mathbf{R}=m \mathbf{a}_{1}+n \mathbf{a}_{2}$ are the lattice sites spanned by the 
vectors

$$
\mathbf{a}_{1}=\frac{L}{2} \mathbf{e}_{x}-\frac{\sqrt{3} L}{2 \alpha} \mathbf{e}_{y}, \quad \mathbf{a}_{2}=\frac{L}{2} \mathbf{e}_{x}+\frac{\sqrt{3} L}{2 \alpha} \mathbf{e}_{y},
$$

where the parameter $\alpha$ represents an uniaxial 'strain' in the $y$ (armchair) direction. For $\alpha<1$, the lattice is prolonged in the $y$ direction and for $\alpha>1$ it is compressed. In the case $\alpha=1$, we obtain the unstrained triangular lattice vectors (1). The corresponding reciprocal lattice to (9) is $\mathbf{G}=k \mathbf{a}_{1}^{*}+l \mathbf{a}_{2}^{*}$, where

$$
\mathbf{a}_{1}^{*}=\frac{2 \pi}{L} \mathbf{e}_{x}-\frac{2 \pi \alpha}{\sqrt{3} L} \mathbf{e}_{y}, \quad \mathbf{a}_{2}^{*}=\frac{2 \pi}{L} \mathbf{e}_{x}+\frac{2 \pi \alpha}{\sqrt{3} L} \mathbf{e}_{y} .
$$

The Brillouin zone of such a lattice is hexagonal (see Fig. 2(b)), but in contrast to the calculations in section II, it is not a regular hexagon unless $\alpha=1$. Its side lengths are

$$
\left|\mathbf{K}_{1}-\mathbf{K}_{2}^{\prime}\right|=\frac{2 \pi \alpha}{\sqrt{3} L} \sqrt{1+\frac{\alpha^{2}}{3}}, \quad\left|\mathbf{K}_{1}-\mathbf{K}_{1}^{\prime}\right|=\frac{2 \pi}{L}\left[1-\frac{\alpha^{2}}{3}\right]
$$

and the positions of $\mathrm{K}_{1}$ and $\mathrm{K}_{1}^{\prime}$ points are

$$
k_{x}^{\mathrm{K}_{1}}=\frac{\pi}{L}\left[1+\frac{\alpha^{2}}{3}\right], \quad k_{x}^{\mathrm{K}_{1}^{\prime}}=\frac{4 \pi}{L}-k_{x}^{\mathrm{K}_{1}} .
$$

We would like to stress that the uniaxial constant strain applied to the antidot lattice does not act as a constant strain on the underlying hexagonal lattice of electrons. For the strain $\alpha=\sqrt{3} \approx 1.73$, the triangular antidot lattice becomes square and the corresponding 'electron' lattice changes from hexagonal to square. A constant strain on the hexagonal lattice would only lead to the deformation of the regular hexagons but would not change the connectivity of the lattice. For $\alpha=\sqrt{3}$ also the Brillouin zone is no longer hexagonal but square and we get $\left|\mathbf{K}_{1}-\mathbf{K}_{1}^{\prime}\right|=0$. For $\alpha>\sqrt{3}$, the antidot lattice becomes the same as the lattice for $\alpha \leq \sqrt{3}$ but it is rotated by $90^{\circ}$ with the rescaled lattice constant $L^{\prime}=\sqrt{3} L / \alpha$ and strain parameter $\alpha^{\prime}=3 / \alpha$. For example, for $\alpha=3$, the antidot lattice again corresponds to the unstrained artificial graphene with $L^{\prime}=L / \sqrt{3}$ and zig-zag orientation in the $y$ direction.

The previous discussion shows, that it is sufficient to restrict the values of $\alpha$ to the interval $[0, \sqrt{3}]$.

\section{B. Numerical model}

The electron wavefunction in artificial graphene is described by the Schrödinger equation

$$
H \psi(\mathbf{r})=\epsilon \psi(\mathbf{r})
$$

with the Hamiltonian

$$
H=-\frac{\hbar^{2}}{2 m^{*}} \Delta+V(\mathbf{r})
$$

where the lattice potential $V(\mathbf{r})$ given by 8$)$ is periodic with the lattice vectors $\mathbf{R}$. Then the electron wavefunctions are of the Bloch form

$$
\psi_{\mathbf{k}}(\mathbf{r})=\sum_{\mathbf{G}} c_{\mathbf{G}}^{\mathbf{k}} e^{i(\mathbf{k}+\mathbf{G}) \cdot \mathbf{r}},
$$

where $\mathbf{k}$ is restricted to the first Brillouin zone. The Schrödinger equation 13 then becomes

$$
\sum_{\mathbf{G}^{\prime}}\left\{\left[\frac{\hbar^{2}(\mathbf{k}+\mathbf{G})^{2}}{2 m^{*}}-\epsilon\right] \delta_{\mathbf{G}, \mathbf{G}^{\prime}}+V_{\mathbf{G}^{\prime}-\mathbf{G}}\right\} c_{\mathbf{G}^{\prime}}^{\mathbf{k}}=0
$$

where the Fourier coefficients of the lattice potential are

$$
V_{\mathbf{Q}}=\frac{1}{S} \int_{\text {cell }} V(\mathbf{r}) e^{i \mathbf{Q} \cdot \mathbf{r}} d \mathbf{r}=\frac{2 \pi \mathcal{V}}{S} \frac{\rho}{Q} J_{1}(Q \rho),
$$

where $J_{1}$ is the Bessel function of the first kind and $S=$ $\sqrt{3} L^{2} /(2 \alpha)$ is the area of the unit cell. Eq. (17) shows that due to the rotational symmetry of an antidot, the Fourier coefficients of $V(\mathbf{r})$ depend only on the length of the wavevector Q. Eq. 16 shows that the periodic lattice potential mixes only those plane waves with wavevectors that are shifted by reciprocal lattice vectors G. Solving (16) numerically we get the complete band structure $\epsilon_{n}(\mathbf{k})$.

In general, the number of terms in the sum (15) is infinite (and the number of the corresponding equations (16) as well) but in practice it is sufficient to assume a finite number of reciprocal lattice vectors $\mathbf{G}$. Because we are interested in the lowest energy bands $\epsilon_{n}(\mathbf{k})$, the most important terms in (15) are the plane waves with the smallest wavevectors. Therefore, we restrict the summation in (15) to the wavevector $\boldsymbol{\Gamma}=\mathbf{0}$ plus a few hexagonal shells of reciprocal lattice points centered around $\boldsymbol{\Gamma}$. The calculated energy bands saturate quickly with increasing number of hexagonal shells $N$ and a sufficient value of $N$ depends on the strength of potential. In our calculations, we use mostly $N=20$.

It can be easily shown that the electron eigenenergies in (16) scale as $\hbar^{2} /\left(m^{*} L^{2}\right)$ and therefore, we introduce the energy unit

$$
E_{0}=\frac{\hbar^{2}}{2 m^{*}}\left(\frac{4 \pi}{3 L}\right)^{2}
$$

which corresponds to the free electron energy at the $\mathrm{K}$ point. Then, we use the dimensionless parameters $E / E_{0}, \mathcal{V} / E_{0}$ and $\rho / L$.

\section{NUMERICAL RESULTS}

In this section, we present the results of our numerical calculations describing the bandstructure of artificial graphene under uniaxial strain. Firstly, we show the results for the unstrained case $\alpha=1$.

\section{A. Unstrained artificial graphene}

It was shown before ${ }^{8}$ that the two lowest energy bands of artificial graphene are similar to the bandstructure of normal 


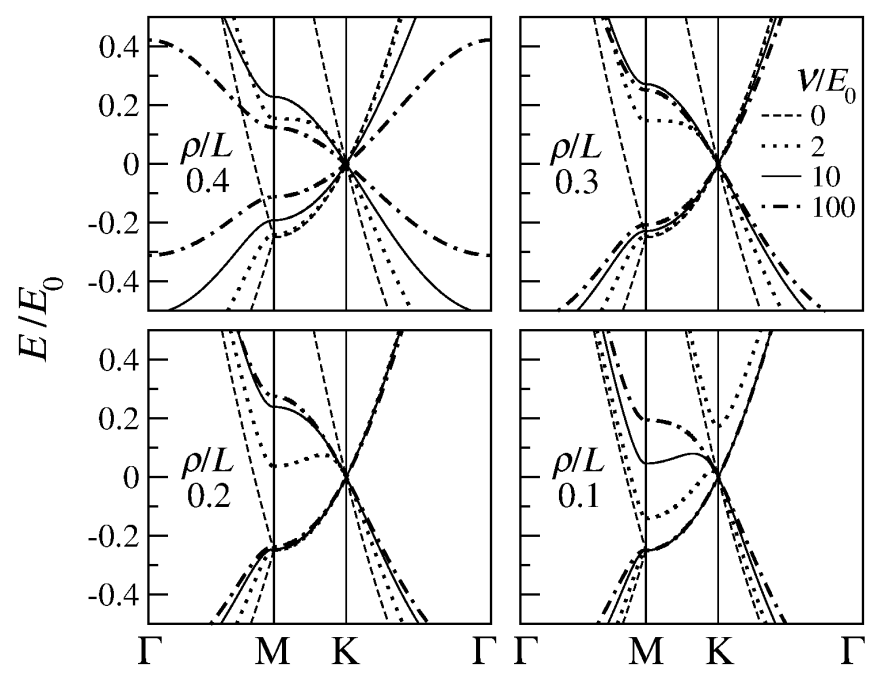

FIG. 3: Bandstructure of the unstrained ( $\alpha=1)$ artificial graphene for various values of antidot radii $\rho$ and strengths $\mathcal{V}$ of the repulsive potential. The bands are shifted by a constant energy to set the energy at the Dirac point equal to zero.

graphene. They touch each other at two Dirac points located at the corners $\mathrm{K}$ of the hexagonal Brillouin zone. In the vicinity of the Dirac points, the bands have linear dispersion and form Dirac cones. Our numerical calculations are in accordance with these results as shown in Fig. 3 for a wide range of potential parameters. The four graphs correspond to various values of antidot radii $\rho$ and each graph contains data for three values of potential strength $\mathcal{V}$. The free-electron bandstructure (dashed lines) is shown for comparison. Let's focus on the two lowest energy bands in the M-K direction. One can observe that when applying the antidot potential, the two lowest energy bands (which are doubly-degenerated for $\mathcal{V}=0$ ) repel each other except at the $\mathrm{K}$ point where the Dirac cone is formed. This process was analytically described in Ref. 8 for small potential strengths in the nearly-free electron (NFE) approximation and its generalization for the strained artificial graphene is presented in the following section.

Figure 4 (a) shows the ratio of the numerically calculated Fermi velocity $v_{F}$ (the slope of the bands crossing at $\mathrm{K}$ ) and the NFE prediction ${ }^{8} v_{0}=2 \pi \hbar /\left(3 m^{*} L\right)$ as a function of the potential strength for various antidot radii.

As expected, the NFE approximation is quite accurate for small values of the potential strength independent of $\rho / L$. With increasing potential strength, the Fermi velocity decreases, an effect that is more pronounced for larger antidots. In the limit $\mathcal{V} / E_{0} \rightarrow \infty$, the Fermi velocity saturates at a constant value which is relatively close to $v_{0}$, for $\rho / L \leq 0.3$.

In order to have Dirac cones as the sole energy values in the vicinity of the Dirac points, the energy gap at the M point (see Fig. (3) should be large enough. This energy gap increases with increasing potential strength and for $\mathcal{V}>\mathcal{V}_{\min }$, the value of the second band at the point $\mathrm{M}$ is higher than the Dirac point. Then, the Dirac cones are the only energy values around Dirac points. Fig. 4(b) shows the minimal value of the potential strength $\mathcal{V}_{\min }$ as a function of the antidot radius. For $\rho / L>$
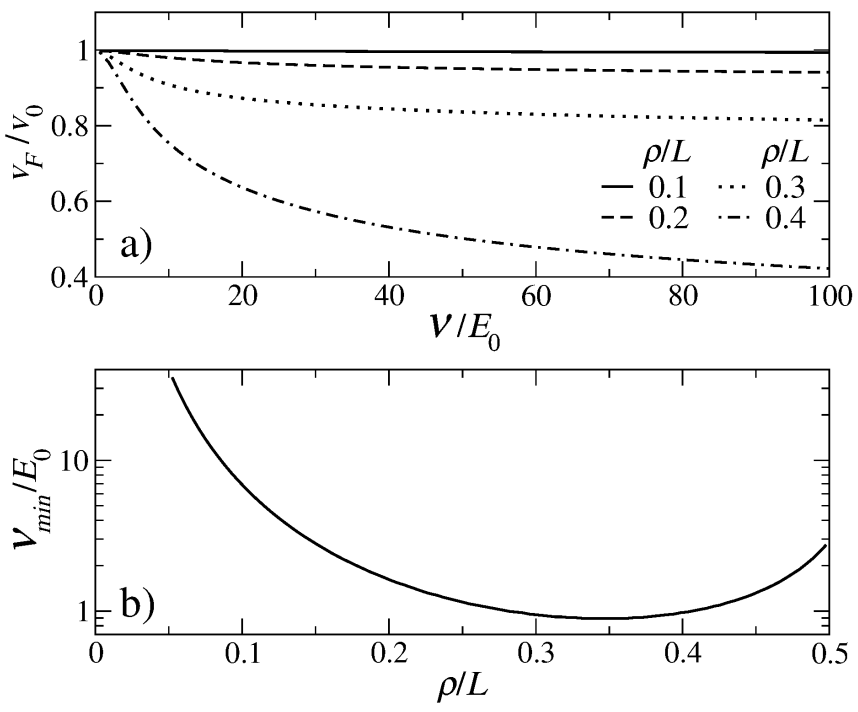

FIG. 4: (a) Ratio of the numerically calculated Fermi velocity $v_{F}$ and the NFE value $v_{0}$ as a function of the potential strength for various antidot radii $\rho / L$. (b) Minimum value of potential strength $\mathcal{V}_{\min }$ to obtain only Dirac cone energies around the Dirac point as a function of antidot radius $\rho / L$.

0.1 , the minimal value of the potential is of the order of $E_{0}$ but for very small $\rho / L$ the potential must be much stronger.

\section{B. Artificial graphene under uniaxial strain}

As was shown in section II, the tight-binding model on a hexagonal lattice displays Dirac points at the corners of the Brillouin zone $\mathrm{K}_{1}$ and $\mathrm{K}_{1}^{\prime}$. When we apply a hopping anisotropy in the armchair direction, the Dirac points are shifted and lie somewhere at the line $\mathrm{CC}^{\prime}$ (see Fig. 11 b)) depending on the value of anisotropy parameter $t^{\prime} / t$. If $t^{\prime} / t<1$ the Dirac points are located inside the Brillouin zone and for $1 \leq t^{\prime} / t \leq 2$ the Dirac points lie at the edge of the Brillouin zone. For $t^{\prime} / t=2$ both Dirac points merge at the point $S$ and for $t^{\prime} / t>2$, an energy gap appears. The aim of this subsection is to show a similar behavior in the case of electronic artificial graphene.

In normal graphene, the hopping anisotropy is induced by applying a mechanical strain. When the lattice is stretched (or compressed), then $t^{\prime} / t<1$ (or $t^{\prime} / t>1$ ). However, to describe a realistic model of normal graphene, the hexagonal model from section II is not sufficient because the change of the lattice geometry due to the applied strain must be taken into account ${ }^{13,24}$ But the results of the realistic mode $1^{13}$ capture essentially the same features as in the case of the undeformed hexagonal model discussed above.

In the case of electronic artificial graphene, the effect of strain can be obtained by tuning the lattice constant in the armchair direction. According to (9), the values $0<\alpha<1$ correspond to stretching and $1<\alpha$ correspond to compression. Like in normal graphene, the lattice deformations imply the deformations of the Brillouin zone. When $0<\alpha<\sqrt{3}$, 


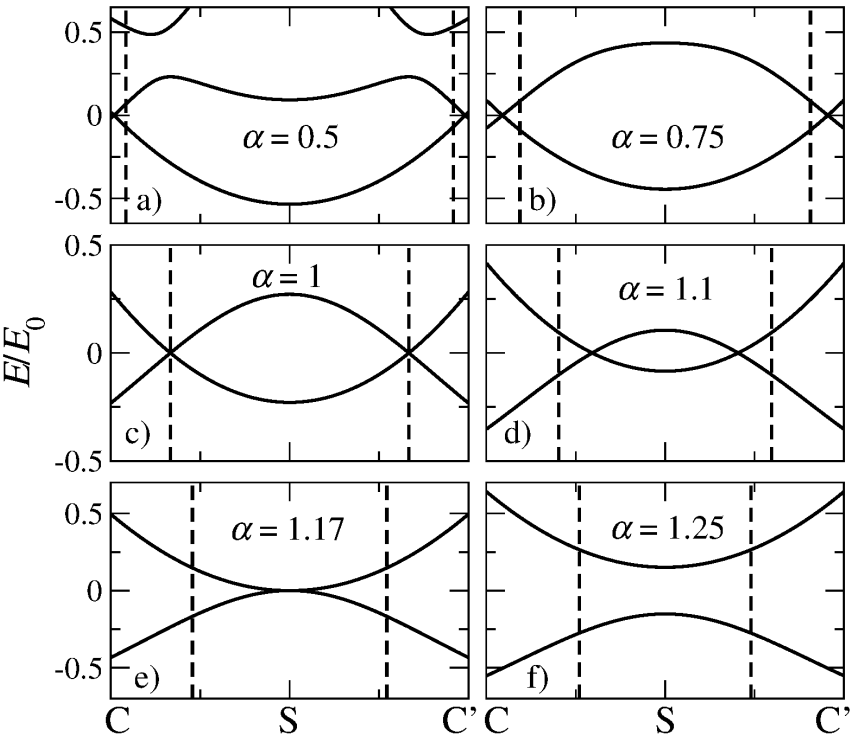

FIG. 5: Bandstructure (solid lines) of artificial graphene changing with the applied uniaxial strain $\alpha$. The bands correspond to the $\mathrm{CC}^{\prime}$ line in the repeated Brillouin zone scheme (see Fig. 2 (b)). Figures (a) and (b) show the case of strained artificial graphene, figure (c) corresponds to the unstrained case and figures (d)-(f) depict the case of compressed artificial graphene. The dashed lines show the positions of the corners of Brillouin zone $K_{1}$ and $K_{1}^{\prime}$ which change with applied strain according to (12). The results are calculated for the potential strength $\mathcal{V} / E_{0}=10$ and antidot radius $\rho / L=0.3$.

the Brillouin zone is hexagonal, but the length of its sides together with the position of the $\mathrm{K}$ points depend on $\alpha$ as shown in (11) and (12).

Figure 5 shows the bandstructure (solid lines) of artificial graphene under uniaxial strain for potential strength $\mathcal{V} / E_{0}=$ 10 and antidot radius $\rho / L=0.3$. For simplicity, we show only the bands on the $\mathrm{CC}^{\prime}$ line which captures the positions of both Dirac points (see Fig. 2(b)). The graphs correspond to various values of strain $\alpha$ and Fig. 5(c) represents the unstrained case where $\alpha=1$. Here, both Dirac points lie at the $\mathrm{K}_{1}$ and $\mathrm{K}^{\prime}{ }_{1}$ points depicted by dashed lines. In the case of stretching (Fig. 5(a), (b)), the Dirac points are located inside the Brillouin zone (outside the $\mathrm{K}_{1} \mathrm{~K}_{1}^{\prime}$ edge) and with increasing stretching (decreasing $\alpha$ ) the Dirac points are shifted towards the ends of $\mathrm{CC}^{\prime}$ line. For $\alpha=0.5$ the Dirac points almost coincide with $\mathrm{C}$ and $\mathrm{C}^{\prime}$. On the other hand, in the case of compressive strain where $\alpha>1$ (Fig. 5)(d)-(f)), the Dirac points lie at the edge $\mathrm{K}_{1} \mathrm{~K}_{1}^{\prime}$ and with increasing compression (increasing $\alpha$ ) they approach each other. For $\alpha=1.17$ the Dirac points merge at the point $\mathrm{S}$ and the dispersion becomes parabolic as shown in Ref. 16. For $\alpha>1.17$, the system undergoes a topological transition, Dirac points no longer exist and the energy gap appears (Fig.5(f)).

To study the positions of the Dirac points as a function of strain, we calculate the distance $\gamma$ of the Dirac points from the $\mathrm{S}$ point given by the formula

$$
\gamma=\frac{\left|\mathbf{D}_{1}-\mathbf{S}\right|}{|\mathbf{C}-\mathbf{S}|}=\frac{\left|\mathbf{D}_{2}-\mathbf{S}\right|}{\left|\mathbf{C}^{\prime}-\mathbf{S}\right|},
$$
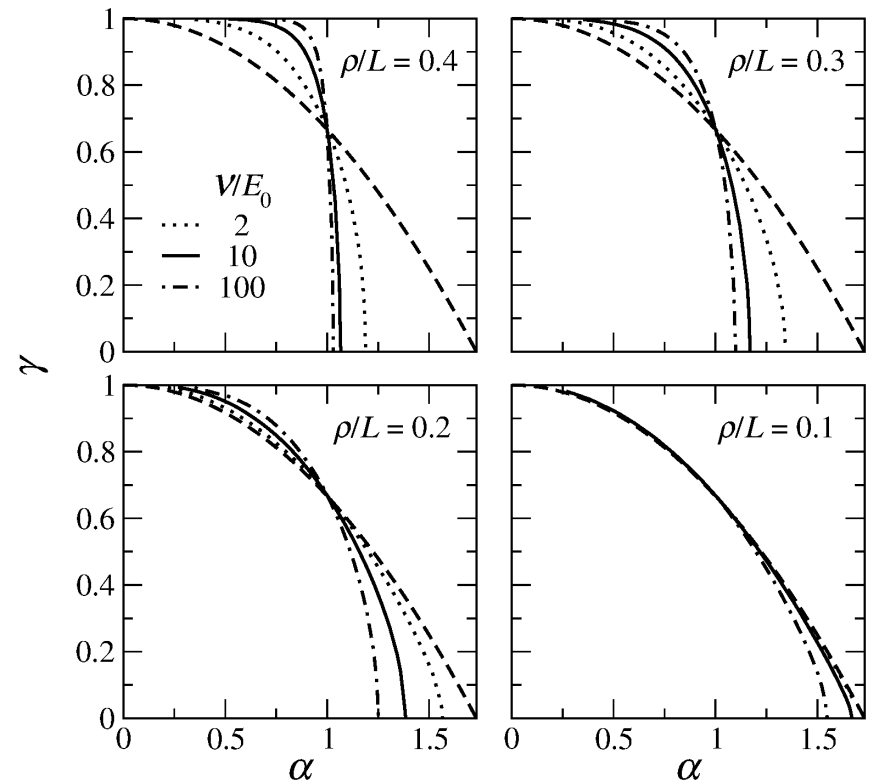

FIG. 6: Relative position of the Dirac points with respect to the Spoint (see Fig. 2 b b) as a function of strain. The graphs correspond to the various antidot radii and contain the data for different potential strengths and these parameters reflect those from Fig. 3. The dashed lines depict the position of the K points given by 201.

where $|\mathbf{C}-\mathbf{S}|=\left|\mathbf{C}^{\prime}-\mathbf{S}\right|=\pi / L$. If $\gamma=1$, the Dirac points lie at $C$ and $C^{\prime}$ and if $\gamma=0$ the Dirac points meet and merge at the $\mathrm{S}$ point.

Figure 6 shows the positions of the Dirac points as a function of strain for four various antidot sizes and three potential strengths corresponding to the parameters in Fig. 3. The dashed lines show the position of the points $K_{1}$ and $K_{1}^{\prime}$ with respect to $S$ given by 12 as

$$
\gamma_{K}=\frac{\left|\mathbf{K}_{1}-\mathbf{S}\right|}{|\mathbf{C}-\mathbf{S}|}=\frac{\left|\mathbf{K}_{1}^{\prime}-\mathbf{S}\right|}{\left|\mathbf{C}^{\prime}-\mathbf{S}\right|}=1-\frac{\alpha^{2}}{3} .
$$

For $\alpha=\sqrt{3} \sim 1.73$, the points $\mathrm{K}_{1}, \mathrm{~K}_{1}^{\prime}$, S merge $\left(\gamma_{K}=0\right)$ and the antidot lattice becomes square instead of triangular.

Figure 6 confirms that the behavior described in Fig. 5 is universal: In the case of stretched artificial graphene $(\alpha<1)$, the Dirac points are located inside the Brillouin zone with the asymptotic position at $\mathrm{C}$ and $\mathrm{C}^{\prime}$ for $\alpha \rightarrow 0$. In the case of zero strain $(\alpha=1)$ the Dirac points lie at $\mathrm{K}_{1}$ and $\mathrm{K}_{1}^{\prime}$ with $\gamma=2 / 3$. When the strain is compressive $(\alpha>1)$, the Dirac points are located at the edge $\mathrm{K}_{1} \mathrm{~K}_{1}^{\prime}$ and move towards each other. At a critical value of strain parameter $\alpha_{c}$, the Dirac points merge at S. For $\alpha>\alpha_{C}$ the energy gap increases with increasing strain.

Figure 7 shows how $\alpha_{C}$ depends on the antidot radius for various values of potential strength. For large and strong potential, $\alpha_{C}$ only slightly differs from 1 which means that the artificial graphene undergoes the topological transition when applying very small compressive strain. On the other hand, in the limit of small antidots, the critical strain parameter is close to $\sqrt{3}$ for arbitrary potential strength. This means that the Dirac points are always located at the corners of the Bril- 


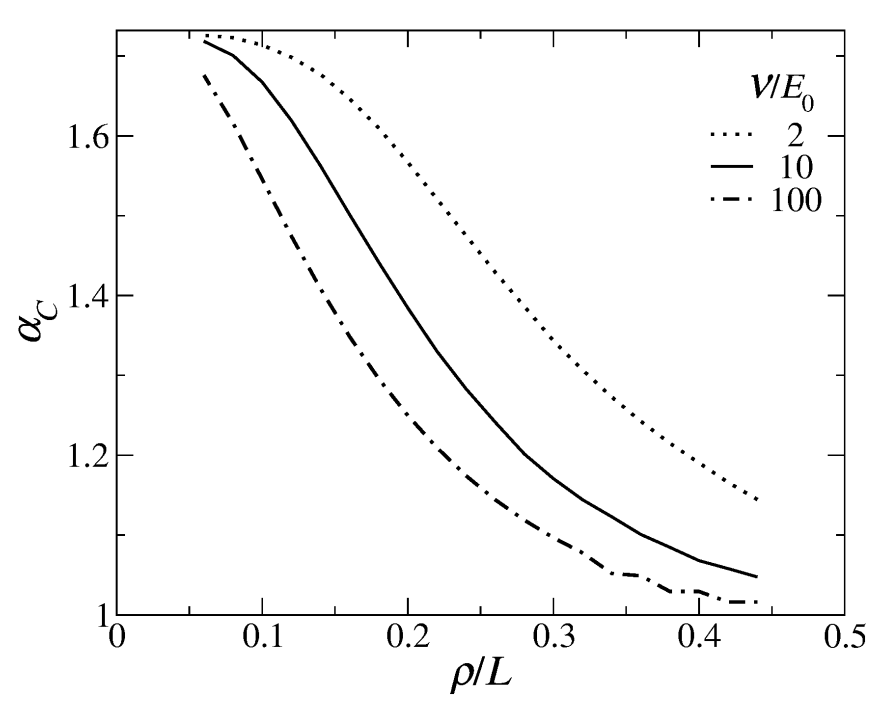

FIG. 7: Critical strain $\alpha_{C}$ corresponding to the merging of the Dirac points as a function of the antidot radius for various values of potential strength $\mathcal{V}$.

louin zone and the system undergoes the topological transition from a conductor into an insulator together with the transition from triangular to square lattice.

\section{ANALYTICAL MODEL FOR WEAK POTENTIALS}

In this section our goal is to explain analytically the results from the previous chapter on the basis of the NFE approach developed in Ref. 8. To calculate a first order correction to the free-electron energies, the potential has to be sufficiently small. Therefore first we examine the validity of the NFE approach with respect to the potential parameters.

For simplicity, we assume the unstrained artificial graphene, where the Dirac points are located at K-points and the Brillouin zone has a shape of a regular hexagon (the same as in Fig. 1(b)). We are interested in the lowest energy band around the Dirac point located at the point $\mathrm{K}_{1}$. In the NFE approach, the lowest-energy electron wavefunction (15) for $\mathbf{k}$ close to $\mathrm{K}_{1}$ can be approximated as a sum of three plane waves $e^{i\left(\mathbf{k}-\mathbf{G}_{j}\right) \cdot \mathbf{r}}$, where $\mathbf{G}_{j}=\left\{\mathbf{0}, \mathbf{a}_{1}^{*}, \mathbf{a}_{2}^{*}\right\}$ with free-electron energies close to $E_{0}$ given by (18). The next most significant plane waves in the expansion (15) would have energies close to $4 E_{0}$. Therefore, the NFE approach is valid when

$$
\left|V_{\mathbf{G}_{j}}\right| \ll 4 E_{0}-E_{0},
$$

where $V_{\mathbf{G}}$ is given by (17). Condition (21) can be rearranged to

$$
\sigma \frac{4 \pi}{\sqrt{3}} \frac{1}{G_{j} \rho} J_{1}\left(G_{j} \rho\right) \ll 3
$$

where

$$
\sigma=\frac{\mathcal{V}}{E_{0}}\left(\frac{\rho}{L}\right)^{2}
$$

is a dimensionless antidot volume. We can replace the term $J_{1}\left(G_{j} \rho\right) /\left(G_{j} \rho\right)$ in 22 by its maximum value 0.5 (for $\rho / L \rightarrow$ 0 ) and finally obtain the condition for the validity of the NFE approximation in the form

$$
\sigma \ll \frac{3 \sqrt{3}}{2 \pi} \sim 1
$$

\section{A. Dirac points}

Now we are about to derive the formula for the position $\gamma$ of the Dirac points as a function of strain.

According to the NFE approach ${ }^{8}$ we can express the electron wavefunction for $\mathrm{k}$ in the vicinity of the Dirac point $\mathrm{D}_{1}$ (see Fig.2(b)) in the basis

$$
|1\rangle=\frac{e^{i \mathbf{D}_{1} \cdot \mathbf{r}}}{\sqrt{S}}, \quad|2\rangle=\frac{e^{i\left(\mathbf{D}_{1}-\mathbf{a}_{1}^{*}\right) \cdot \mathbf{r}}}{\sqrt{S}}, \quad|3\rangle=\frac{e^{i\left(\mathbf{D}_{1}-\mathbf{a}_{2}^{*}\right) \cdot \mathbf{r}}}{\sqrt{S}},
$$

as

$$
\psi(\mathbf{r})=e^{i \mathbf{q} \cdot \mathbf{r}}\left[b_{1}|1\rangle+b_{2}|2\rangle+b_{3}|3\rangle\right],
$$

where we have used $\mathbf{k}=\mathbf{q}+\mathbf{D}_{1}$ and

$$
\mathbf{D}_{1}=\frac{\mathbf{a}_{2}^{*}+\mathbf{a}_{1}^{*}}{2}\left(1-\frac{\gamma}{2}\right)
$$

is the position vector of the Dirac point $\mathrm{D}_{1}$. Then we can write the Hamiltonian (14) in the basis (25) as

$$
H=H_{0}+H_{1}
$$

where

$$
H_{0}=\left(\begin{array}{ccc}
\frac{\hbar^{2}}{2 m^{*}}\left|\mathbf{D}_{1}\right|^{2} & V_{\mathbf{a}_{1}^{*}} & V_{\mathbf{a}_{2}^{*}} \\
V_{-\mathbf{a}_{1}^{*}} & \frac{\hbar^{2}}{2 m^{*}}\left|\mathbf{D}_{1}-\mathbf{a}_{1}^{*}\right|^{2} & V_{\mathbf{a}_{2}^{*}-\mathbf{a}_{1}^{*}} \\
V_{-\mathbf{a}_{2}^{*}} & V_{\mathbf{a}_{1}^{*}-\mathbf{a}_{2}^{*}} & \frac{\hbar^{2}}{2 m^{*}}\left|\mathbf{D}_{1}-\mathbf{a}_{2}^{*}\right|^{2}
\end{array}\right)
$$

and for $q L \ll 1$

$H_{1}=\frac{\hbar^{2}}{2 m^{*}}\left(\begin{array}{ccc}2 \mathbf{q} \cdot \mathbf{D}_{\mathbf{1}} & 0 & 0 \\ 0 & 2 \mathbf{q} \cdot\left(\mathbf{D}_{1}-\mathbf{a}_{1}^{*}\right) & 0 \\ 0 & 0 & 2 \mathbf{q} \cdot\left(\mathbf{D}_{\mathbf{1}}-\mathbf{a}_{2}^{*}\right)\end{array}\right)$

In (29), we have omitted the diagonal terms $V_{\mathbf{0}}$ which only cause a shift of the energy eigenvalues. Because $\left|\mathbf{a}_{1}^{*}\right|=\left|\mathbf{a}_{2}^{*}\right|$ and $\left|\mathbf{D}_{1}-\mathbf{a}_{1}^{*}\right|=\left|\mathbf{D}_{1}-\mathbf{a}_{2}^{*}\right|$ the Hamiltonian $H_{0}$ can be written in the form

$$
H_{0}=\left(\begin{array}{ccc}
W_{1} & A_{1} & A_{1} \\
A_{1} & W_{2} & A_{2} \\
A_{1} & A_{2} & W_{2}
\end{array}\right)
$$

where $A_{1}=V_{\mathbf{a}_{1}^{*}}=V_{-\mathbf{a}_{1}^{*}}=V_{\mathbf{a}_{2}^{*}}=V_{-\mathbf{a}_{2}^{*}}, A_{2}=V_{\mathbf{a}_{1}^{*}-\mathbf{a}_{2}^{*}}=$ $V_{\mathbf{a}_{2}^{*}-\mathbf{a}_{1}^{*}}, W_{1}=\frac{\hbar^{2}}{2 m^{*}}\left|\mathbf{D}_{1}\right|^{2}$ and $W_{2}=\frac{\hbar^{2}}{2 m^{*}}\left|\mathbf{D}_{1}-\mathbf{a}_{1}^{*}\right|^{2}=$ $\frac{\hbar^{2}}{2 m^{*}}\left|\mathbf{D}_{\mathbf{1}}-\mathbf{a}_{2}^{*}\right|^{2}$.

We are searching for the position of the Dirac point $D_{1}$ and therefore we expect that for $\mathbf{q}=0$ two energy bands touch at 
$D_{1}$. This means that the Hamiltonian $H(\mathbf{q}=0)=H_{0}$ should have a pair of equal eigenvalues. The eigenvalues of (31) are

$$
\begin{aligned}
& \epsilon_{1}=-A_{2}+W_{2}, \\
& \epsilon_{2}=\frac{1}{2}\left(A_{2}+W_{1}+W_{2}-\sqrt{8 A_{1}^{2}+\left(A_{2}+W_{2}-W_{1}\right)^{2}}\right), \\
& \epsilon_{3}=\frac{1}{2}\left(A_{2}+W_{1}+W_{2}+\sqrt{8 A_{1}^{2}+\left(A_{2}+W_{2}-W_{1}\right)^{2}}\right) .
\end{aligned}
$$

We assume that the first and second eigenvalue are equal which yields the condition

$$
A_{1}^{2}-A_{2}^{2}+A_{2}\left(W_{2}-W_{1}\right)=0 .
$$

This condition determines the position $\gamma$ of the Dirac point $D_{1}$ which is hidden in the diagonal terms $W_{1}$ and $W_{2}$ in the form

$$
W_{2}-W_{1}=\frac{\hbar^{2}}{2 m^{*}}\left(\frac{2 \pi}{L}\right)^{2}\left[\gamma+\frac{\alpha^{2}}{3}-1\right] .
$$

We first explore the limit $\rho / L \ll 1$. In this case the Fourier coefficients $V_{\mathbf{p}}$ given by (17) can be simplified as

$$
V_{\mathbf{Q}}=\frac{2 \pi \mathcal{V}}{S} \frac{\rho}{Q} J_{1}(Q \rho) \sim \frac{2 \pi \mathcal{V}}{S} \frac{\rho}{Q}\left(\frac{Q \rho}{2}\right)=\frac{\pi \rho^{2} \mathcal{V}}{S}=V_{\mathbf{0}}
$$

which is independent on the wavevector $\mathbf{Q}$. Therefore $A_{1}=$ $A_{2}$ and from (32) we get $W_{1}=W_{2}$. Then, from (33) we get $\gamma=1-\alpha^{2} / 3$ which is exactly the same as (20). This means that for $\rho / L \ll 1$ the Dirac point $D_{1}$ is located directly at the corner $K_{1}$ of the Brillouin zone. This explains the behavior observed in Fig. 6, where with decreasing antidot radius the position of Dirac points is closer to the dashed line given by (20). For $\rho / L=0.1$ and $\mathcal{V} / E_{0}=2$, the position of Dirac points and K-points is indistinguishable. Then the critical strain $\alpha_{C}$, which leads to the merging of the Dirac points, is equal to the strain corresponding to the transition from triangular to square antidot lattice, i.e., $\alpha_{C}=\sqrt{3}$.

Now we put the expressions (17) and (31) into (32) and obtain $\gamma$ as a function of strain $\alpha$ and relative barrier size $\rho / L$ in the form

$$
\begin{gathered}
\gamma=1-\frac{\alpha^{2}}{3}+\sigma \frac{4}{9} \frac{L}{\rho}\left\{J_{1}\left[\frac{4 \pi \alpha}{\sqrt{3}} \frac{\rho}{L}\right]-\right. \\
\left.\frac{4 \alpha^{2}}{\alpha^{2}+3} \frac{J_{1}^{2}\left[2 \pi \sqrt{1+\frac{\alpha^{2}}{3}} \frac{\rho}{L}\right]}{J_{1}\left[\frac{4 \pi \alpha}{\sqrt{3}} \frac{\rho}{L}\right]}\right\},
\end{gathered}
$$

where $\sigma$ is the dimensionless volume of the barrier given by (23).

Fig. 8 shows the position of the Dirac points $\gamma$ as a function of the strain parameter $\alpha$ for weak potentials with the antidot volume $\sigma=0.08$. The numerical data (filled symbols) and corresponding analytical formula (35) (solid lines) are plotted for various antidot radii shown in the legend. The analytical formula roughly agrees with the numerical data except for the values of $\alpha$ in the vicinity of critical value $\alpha_{C}$. Here, the NFE basis (25) is not sufficient because there exists another plane wave with similar energy (for $\mathbf{G}=\mathbf{a}_{1}^{*}+\mathbf{a}_{2}^{*}$ ).

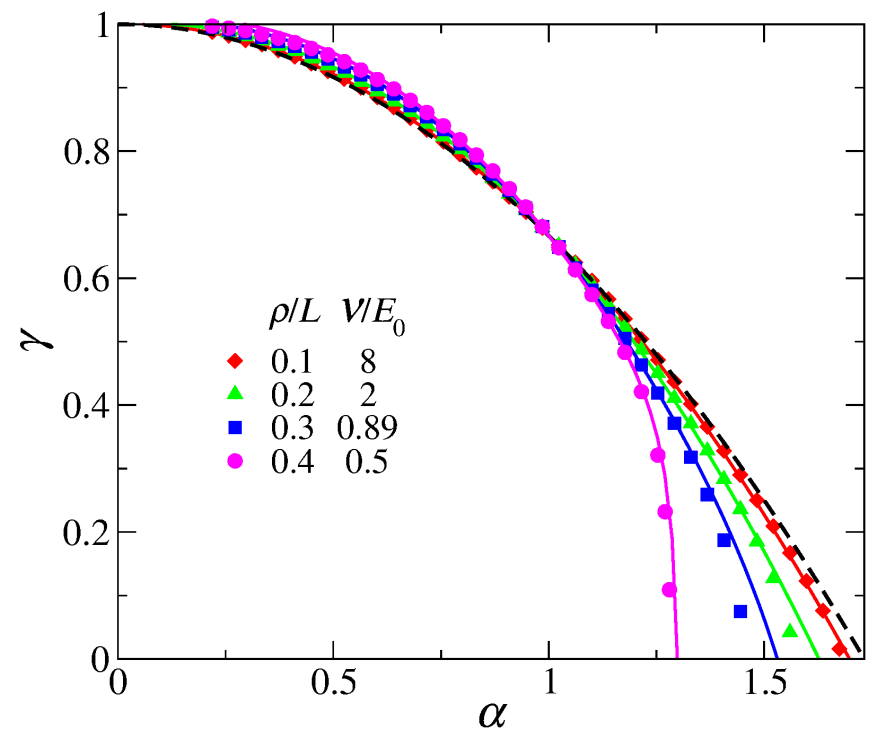

FIG. 8: Position of the Dirac points with respect to the point $S$ as a function of the strain parameter for weak potentials with constant antidot volume $\sigma=0.08$ and various radii listed in the legend. The numerical data are shown as filled symbols and solid lines correspond to the analytical formula (35). The dashed line represents the position of the K-point given by 20 .

\section{ABOUT THE EXPERIMENTAL REALIZABILITY}

In experiments with artificial graphene, $\frac{3,4}{4}$ the merging of the Dirac points is usually revealed when measuring the density of states (or some related quantity). When the Dirac points exist, the DOS is gapless and equal zero at the energy of the Dirac point. When the Dirac points merge, the energy gap opens which is visible also in the DOS. In this section, we propose the experimental observation of Dirac point merging in the electronic systems based on the artificial graphene studied in this paper.

We first discuss the conditions under which the energy gap in the DOS opens as a result of Dirac point merging. To observe the energy gap in the DOS, no electron energies should lie in the energy window given by the gap between the bands. Otherwise, the energy gap in the DOS would not open even if the Dirac points merged. To analyze this problem, it is sufficient to study the bandstructure at the edges of the Brillouin zone, because here the maximum of the first band and minimum of the second band are located. Fig. 9 shows how these bands change with increasing compressive strain. The graphs represent the energy bands corresponding to the $\mathrm{MK}_{1} \mathrm{~S}$ line at the edge of the Brillouin zone (see Fig.2(b)) calculated for antidot radius $\rho / L=0.2$ and two values of the potential strength $\mathcal{V} / E_{0}=3$ (solid lines) and $\mathcal{V} / E_{0}=6$ (dashed lines).

Fig. 9 a) shows the data for unstrained artificial graphene. According to Fig. 4(b), both potential strengths satisfy the condition $\mathcal{V}>\mathcal{V}_{\text {min }}$ which means that a pair of Dirac cones located at $\mathrm{K}_{1}$ and $\mathrm{K}_{1}{ }_{1}$ (not shown here) are the sole energy values in the vicinity of Dirac point. With increasing compression (Figs. 9(b)-(d)), one can observe at the right part of each panel that the Dirac point moves to the point $\mathrm{S}$ where it 

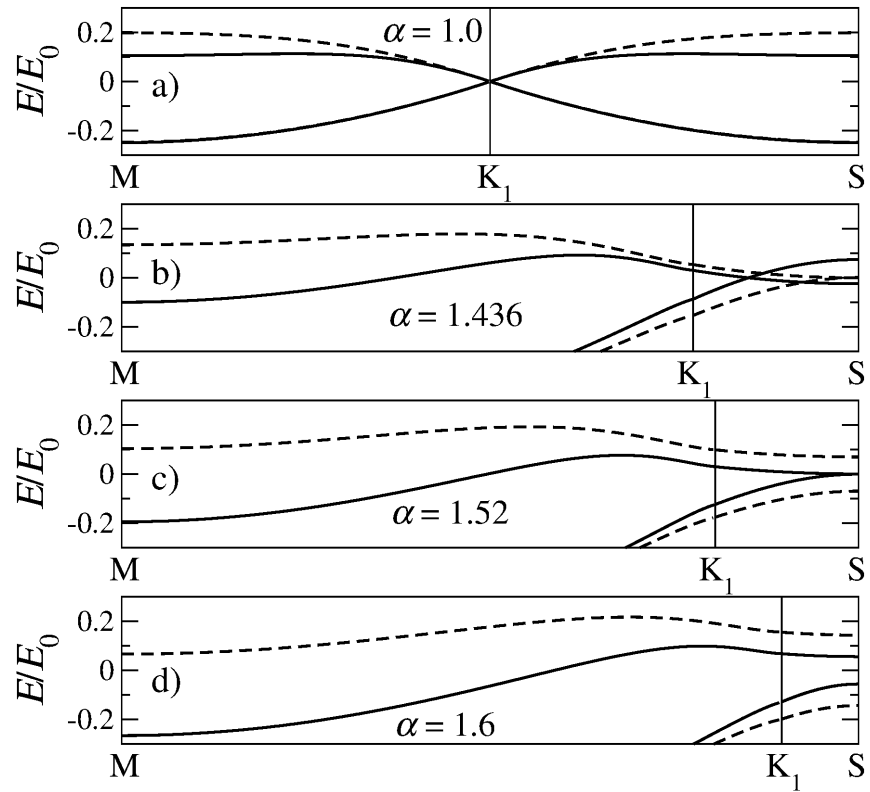

FIG. 9: The development of the bandstructure of artificial graphene with increasing compression given by the strain parameter $\alpha$ shown in the legend. The bands correspond to the line $\mathrm{MK}_{1} \mathrm{~S}$ in the Brillouin zone (see Fig. 2(b)) where the maximum of the first band and the minimum of the second band are located. The parameters are $\rho / L=0.2$ and $\mathcal{V} / E_{0}=3$ (solid lines) or $\mathcal{V} / E_{0}=6$ (dashed lines). Graph (a) shows the bands of unstrained artificial graphene where the Dirac cones are the only energy values around the Dirac points. Graphs (b) and (c) depict the merging of the Dirac point for $\mathcal{V} / E_{0}=6$ and $\mathcal{V} / E_{0}=3$, respectively. The graph (d) shows the situation where the Dirac points are already merged and the gap between the bands exists. In the case of $\mathcal{V} / E_{0}=6$, the maximum of the first band is lower than the minimum of the second band, which means that the gap appears also in the DOS. In the case of $\mathcal{V} / E_{0}=3$, the gap between the bands is obscured by the second energy band and the DOS remains gapless.

merges with the second Dirac point (not shown) and the gap opens. At the same time, the energies of the second band in the left part of the panels are getting lower with respect to the Dirac point. When the Dirac points merge for $\mathcal{V} / E_{0}=3$ (Fig. 9(c)), the energy minimum of the second band located at the $\mathrm{M}$ point is already lower than the Dirac point. Therefore, when the gap in S opens (Fig. 9(d)), it does not appear in the the DOS because it is obscured by the second energy band. On the other hand, in the case of stronger potential $\mathcal{V} / E_{0}=6$ when the Dirac points merge (Fig. Q(b)), the energies of the second band are well above the Dirac point. When the energy gap opens (Figs. 9(c,d)), the energies of the second band are still higher than the maximum of the first band located at the point $\mathrm{S}$. This means that the energy gap is visible also in the DOS.

The above analysis of the bandstructure has revealed that for every $\rho / L$ there is a minimal value of potential strength $\mathcal{V}_{\min }^{\text {DOS }}$ that is necessary to observe the gap opening in the DOS and this value is larger than $\mathcal{V}_{\text {min }}$ given by Fig. $4($ b). We obtain $\mathcal{V}_{\min }^{\text {DOS }}$ numerically using the constraint that for critical strain $\alpha_{C}^{\mathrm{DOS}}$ when the Dirac points merge, the energy value of the
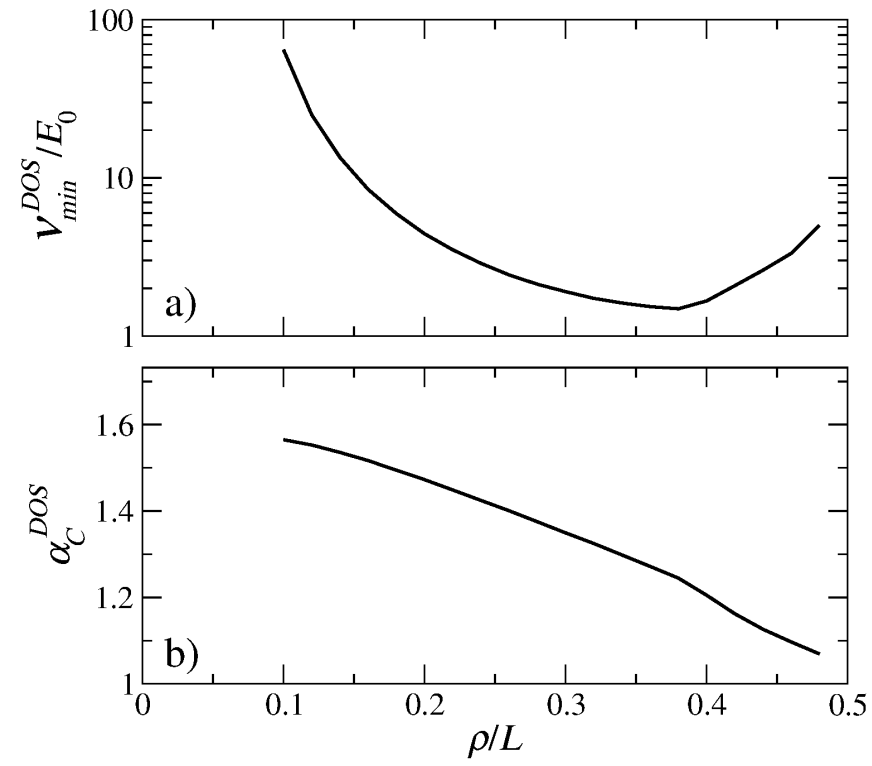

FIG. 10: (a) The minimal value of the potential strength $\mathcal{V}_{\text {min }}^{\mathrm{DOS}}$ that is needed to observe the gap in the DOS. This value is calculated as a function of the normalized antidot radius $\rho / L$. To obtain $\mathcal{V}_{\min }^{\mathrm{DOS}}$, two conditions have to be satisfied simultaneously. Firstly, the Dirac points merge and the bands touch each other only at the point S. Secondly, the minimum of the second energy band, which is located at the point M (see Fig.9), should be equal to the energy of the Dirac point. This means that for $\mathcal{V}=\mathcal{V}_{\min }^{\mathrm{DOS}}$ the minimum of the second energy band is equal to the maximum of the first band. (b) Critical strain corresponding to the potential with the strength $\mathcal{V}_{\min }^{\mathrm{DOS}}$.

second band at the point $\mathrm{M}$ should be equal to the energy of the Dirac point. Then, for the potentials larger than $\mathcal{V}_{\min }^{\mathrm{DOS}}$ there is an interval of strain parameter $\alpha$ where the gap in the DOS appears. For $\mathcal{V}<\mathcal{V}_{\min }^{\mathrm{DOS}}$ the gapped DOS cannot be observed regardless of the value of strain $\alpha$. Fig. 10 shows the calculated $\mathcal{V}_{\min }^{\mathrm{DOS}}$ together with the corresponding critical strain $\alpha_{C}^{\mathrm{DOS}}$.

There have been a couple of attempts to observe the Dirac quasiparticles in the experimental electronic systems based on the theoretical model of artificial graphene studied in this paper. In the work of Ref. 10, the authors discussed a possible realization of artificial graphene by the two-dimensional electron gas (2DEG) in a semiconductor heterostructure where the triangular lattice of antidots is created by etching or gating. Moreover, they performed an experiment with a $2 \mathrm{DEG}$ made from GaAs (with the effective mass of the electrons $m^{*}=0.067 m_{e}$ ) where the antidot lattice was etched with the lattice constant $L=200 \mathrm{~nm}$ and $\rho / L \sim 0.15$. The estimated potential strength was about 2-4 meV. These parameters correspond to $\mathcal{V} / E_{0} \sim 10$ where $E_{0} \sim 0.25 \mathrm{meV}$. Although the presence of Dirac electrons was not fully confirmed in their system (due to the strong disorder) the authors conclude that this should be technologically feasible by reducing the lattice constant of the antidot lattice below $100 \mathrm{~nm}$.

The same concept of artificial graphene was used in the experiments with so-called molecular graphene ${ }^{6,7}$ where the 2DEG on the copper surface is modulated by the triangular 

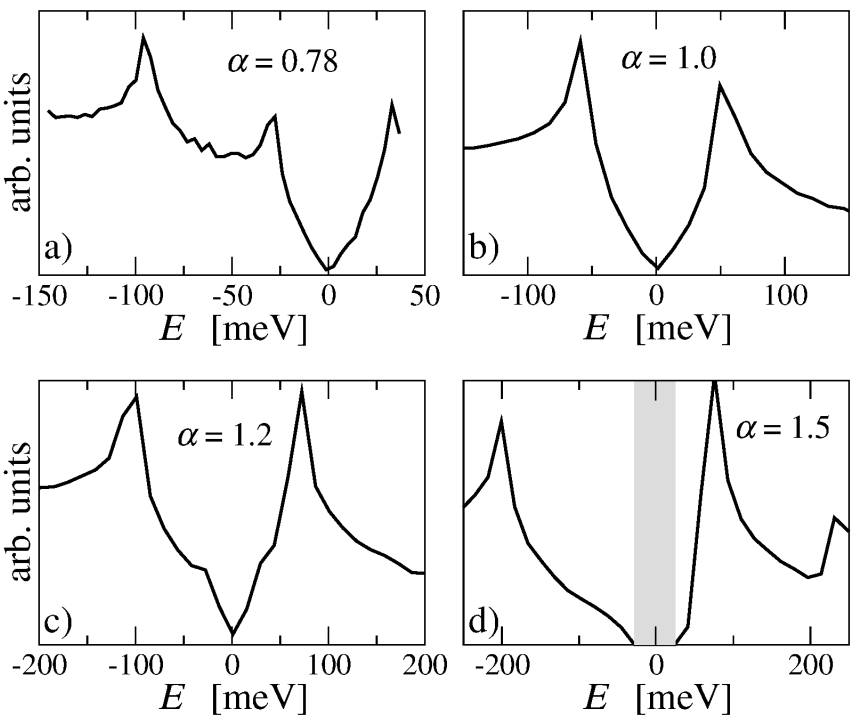

FIG. 11: The DOS of electrons in artificial graphene calculated as a function of the energy for various values of strain parameter $\alpha$ shown in the legend. The parameters $\rho=0.7 \mathrm{~nm}, L=2.7 \mathrm{~nm}$, and $\mathcal{V}=1 \mathrm{eV}$ used in the simulation are the same as in the experiment with molecular graphene created by coronene. ${ }^{7}$ (a) corresponds to the strained artificial graphene, (b) shows the unstrained case, and (c)-(d) corresponds to the artificial graphene compressed in the armchair direction. The gray region in (d) depicts the energy gap created as a result of the merging of the Dirac points.

antidot lattice of repulsive molecules ( $\mathrm{CO}$ or coronene). The manipulation of several hundreds of molecules is provided by a scanning tunneling microscopy. The effective mass of surface electrons is $m^{*}=0.38 m_{e}$ and the lattice constant of the antidot lattice $L=1-3 \mathrm{~nm}$ varied for different experimental situations. The radius of the antidot depends on the details of the electrostatic potential of the repulsive molecule and in the case of coronene it is estimated ${ }^{7}$ to be $\rho=0.7 \mathrm{~nm}$. In the case of $\mathrm{CO}$, the authors of Ref. 6 do not provide the exact value of $\rho$ but it can be roughly estimated from the topographs depicting the distribution of electron density in the modulated 2DEG as $\rho \sim 0.4 \mathrm{~nm}$. A potential strength of an antidot was estimated roughly to be $\mathcal{V} \sim 1 \mathrm{eV}$ for both types of molecules. The presence of Dirac quasiparticles was demonstrated by the measurements of the differential conductance between the STM tip and the $\mathrm{Cu}$ surface that mimic the DOS of modulated electrons. The spatially averaged differential conductance spectrum showed the features typical for the DOS of graphene: a zero value at the Dirac point, linear shape around it and a pair of van Hove singularities.

For the purposes of this paper, particularly interesting is the experiment described in the supporting online material of the work in Ref. 6 where the authors studied the effect of uniaxial strain on $\mathrm{CO}$ molecular graphene. Here, the lattice constant was $L=1.785 \mathrm{~nm}$ which gives $\mathcal{V} / E_{0} \sim 2$ and $\rho / L \sim 0.4$. The measurement of the differential conductance spectrum showed that when the molecular graphene is elongated (stretched) by about $30 \%$ in the armchair direction the DOS around the Dirac point remains linear and gapless.
This is in agreement with our calculations which predict that in the stretched artificial graphene the Dirac points exist and they are shifted inside the Brillouin zone. We would like to stress that according to our results, the merging of the Dirac points appears if the artificial graphene is compressed (instead of stretched) in the armchair direction.

According to Fig. 10 a), the potential strength $\mathcal{V} / E_{0} \sim 2$ at $\rho / L \sim 0.4$ is close to the value $\mathcal{V}_{\min }^{\mathrm{DOS}}$. Therefore, it is not sure that it would be possible to observe the merging of Dirac points in the experiment with $\mathrm{CO}$ molecules. More promising is the molecular graphene made from coronene, $\frac{7}{,}$ where the lattice constant is $L=2.7 \mathrm{~nm}$, which gives $\rho / L=0.26$ and $\mathcal{V} / E_{0}=4$, a value well above $\mathcal{V}_{\text {min }}^{\text {DOS }}$. Fig. 11 shows the DOS of artificial graphene calculated for these experimental parameters. The different graphs show how the DOS changes with strain. Figure 11 b) shows the DOS for unstrained artificial graphene, which is very similar to the DOS of normal graphene. The graph 11 a) corresponds to the situation where the artificial graphene is strained by about $30 \%$ which models the experimental situation discussed above. As expected, the DOS is gapless because the Dirac points do not merge. On the other hand, the graphs 11 (c-d) show how the DOS changes with compression. The merging of the Dirac points occurs for $\alpha_{C}=1.33$, therefore for $\alpha=1.5$ (Fig. 11d)), the gap in the DOS (gray region) is nicely recognizable. The value $\alpha=1.5$ corresponds to the compression of the antidot lattice of about $100(1-1 / \alpha) \sim 33 \%$ which should be possible to realize in the experiment with molecular graphene.

As discussed in this paper, merging of the Dirac points occurs in artificial graphene under uniaxial compression in the armchair direction. We would like to stress that the same effect can be obtained by stretching the antidot lattice in the zigzag (horizontal) direction. In our model, stretching in the zigzag direction just increases the lattice constant $L$ to $L^{\prime \prime}$ while the vertical dimension of the antidot lattice remains the same. Therefore, the antidot lattice stretched in zigzag direction is the same as the lattice with lattice constant $L^{\prime \prime}$ compressed in the armchair direction.

\section{CONCLUSIONS}

In normal graphene, the theory based on tight-binding approximation predicts that with increasing anisotropy in the hopping matrix elements, both Dirac points are moving along the edge of the Brillouin zone towards each other until they merge. This anisotropy can be realized by application of compressive strain in the armchair direction. A merging of the Dirac points was observed so far only in artificial systems that mimic the properties of graphene: in the experiments with confined microwaves in a hexagonal array of waveguides ${ }^{4,5}$ and in a laser lattice with cold atoms $\stackrel{3,18}{3}$

We have shown numerically and analytically that the merging of the Dirac points can be observed also in electronic artificial graphene. The artificial graphene we considered is to be created from the two-dimensional electron gas by applying a repulsive triangular potential and the effect of strain was modeled by reducing the distance between the repulsive potentials 
along the armchair direction.

Our numerical calculations have show that molecular graphene with coronene ${ }^{7}$ is a promising candidate to observe the merging of the Dirac points. This should occur for a lattice compression of about $25 \%$, which appears technologically feasible.
* Electronic address: juraj.feilhauer@ptb.de

1 A. H. Castro Neto, F. Guinea, N. M. Peres, K. S. Novoselov, and A. K. Geim, Rev. Mod. Phys. 81, 109 (2009).

2 M. Polini, F. Guinea, M. Lewenstein, H. C. Manoharan, and V. Pellegrini, Nat. Nanotechnol. 8, 625 (2013).

${ }^{3}$ L. Tarruell, D. Greif, T. Uehlinger, G. Jotzu, and T. Esslinger, Nature 483, 302 (2012).

${ }^{4}$ M. Bellec, U. Kuhl, G. Montambaux, and F. Mortessagne, Phys. Rev. Lett. 110, 033902 (2013).

5 Y. Plotnik et al., Nat. Mater. 13, 57 (2013)

${ }^{6}$ K. K. Gomes, W. Mar, W. Ko, F. Guinea and H. C. Manoharan, Nature 483, 306 (2012) and corresponding supplemental online material.

7 S. Wang, L. Z. Tan, W. Wang, S. G. Louie and N. Lin, Phys. Rev. Lett. 113, 196803 (2014).

${ }^{8}$ C.-H. Park and S. G. Louie, Nano Lett. 9, 1793 (2009).

9 M. Gibertini, A. Singha, V. Pellegrini, M. Polini, G. Vignale, A. Pinczuk, L. N. Pfeiffer, and K. W. West, Phys. Rev. B 79, 241406(R) (2009).

${ }^{10}$ L. Nádvorník, M. Orlita, N. A. Goncharuk, L. Smrčka, V. Novák, V. Jurka, K. Hruška, Z. Výborný, Z. R. Wasilewski, M. Potemski, and K. Výborný, New J. Phys. 14, 053002 (2012).

11 E. Kalesaki, C. Delerue, C. Morais Smith, W. Beugeling, G. Allan, and D. Vanmaekelbergh, Phys. Rev. X 4, 011010 (2014).

12 Y. Hasegawa, R. Konno, H. Nakano, and M. Kohmoto, Phys. Rev.
B 74, 033413 (2006).

13 V. M. Pereira, A. H. Castro Neto, and N. M. R. Peres, Phys. Rev. $B$ 80, 045401 (2009).

14 P. Dietl, F. Piéchon, and G. Montambaux, Phys. Rev. Lett. 100, 236405 (2008).

15 J.-N. Fuchs, arXiv:1306.0380

16 G. Montambaux, F. Piéchon, J.-N. Fuchs, and M. O. Goerbig, Phys. Rev. B 80, 153412 (2009).

17 S.-L. Zhu, B. Wang, and L.-M. Duan, Phys. Rev. Lett. 98, 260402 (2007).

18 B. Wunsch, F. Guinea, and F. Sols, New J. Phys. 10, 103027 (2008).

19 O. A. Tkachenko and V. A. Tkachenko, JETP Lett. 99, 204 (2014).

20 O. P. Sushkov and A. H. Castro Neto, Phys. Rev. Lett. 110, 186601 (2013).

${ }^{21}$ C.-H. Park, L. Yang, Y.-W. Son, M. L. Cohen, and S. G. Louie, Phys. Rev. Lett. 101, 126804 (2008).

${ }^{22}$ M. Aichinger, S. Janecek, I. Kylänpää, and E. Räsänen, Phys. Rev. B 89, 235433 (2014).

23 Z. Liu, J. Wang, and J. Li, Phys. Chem. Chem. Phys. 15, 18855 (2013).

${ }^{24}$ M. R. Masir, D. Moldovan, and F. M. Peeters, Solid State Commun. 175-176, 76 (2013). 